\title{
Real world data of combined lung cancer and interstitial lung disease
}

\author{
Tatsuo Kawahara ${ }^{1}$, Hiroyuki Sakashita ${ }^{1,2}$, Takafumi Suzuki ${ }^{1}$, Tomoya Tateishi $^{1}$, Yasunari Miyazaki ${ }^{1}$ \\ ${ }^{1}$ Department of Respiratory Medicine, Tokyo Medical and Dental University, Tokyo, Japan; ${ }^{2}$ Department of Chemotherapy, Yokosuka Kyosai \\ Hospital, Kanagawa, Japan \\ Contributions: (I) Conception and design: T Kawahara, H Sakashita, Y Miyazaki; (II) Administrative support: Y Miyazaki; (III) Provision of study \\ materials or patients: All authors; (IV) Collection and assembly of data: T Kawahara, H Sakashita; (V) Data analysis and interpretation: T Kawahara, \\ H Sakashita; (VI) Manuscript writing: All authors; (VII) Final approval of manuscript: All authors. \\ Correspondence to: Hiroyuki Sakashita, MD, PhD. Department of Respiratory Medicine, Tokyo Medical and Dental University, 1-5-45, Yushima, \\ Bunkyo-ku, Tokyo 113-8510, Japan. Email: hsakpulm@yahoo.co.jp.
} Background: Research patients with combined lung cancer (LC) and interstitial lung disease (ILD)
is insufficient. The objective of the study was to identify the frequency of such patients and treatments,
including best supportive care (BSC), in the real world.

Methods: All patients with LC who were admitted to our hospital over a 5-year period from 2013 to 2017 were retrospectively analysed.

Results: In this study, 698 patients with LC were enrolled. A total of 148 (21.2\%) suffered from LC and ILD. Seventy-one LC-ILD patients received chemotherapy, and 20 LC-ILD patients received BSC only. Eleven BSC patients whom physicians thought could receive therapy declined treatment. The median survival time of patients who received chemotherapy was 14.3 months (95\% confidence interval, 8.5-19.4 months). The median survival time of BSC patients who declined treatment was 7.2 months and that was shorter than that of patients who received chemotherapy.

Conclusions: This study suggests that patients with combined LC and ILD are common (21.2\%) in the real world, and there is the possibility that therapy for LC-ILD patients is beneficial.

Keywords: Lung cancer (LC); interstitial lung disease (ILD); chemotherapy; best supportive care (BSC)

Submitted Apr 09, 2019. Accepted for publication Sep 28, 2019.

doi: $10.21037 /$ jtd.2019.10.01

View this article at: http://dx.doi.org/10.21037/jtd.2019.10.01

\section{Introduction}

The development of new treatments has gradually improved lung cancer (LC) mortality in this decade. However, patients with lung cancer combined with interstitial lung disease (ILD) (LC-ILD) are not able to fully benefit from these new therapies. In most clinical trials, LC-ILD patients are excluded. Researchers regard the frequency of LCILD patients to be rare, and LC-ILD patients as high-risk targets. Moreover, real-world data about the treatment and risk of treatment-related acute exacerbation (AE) of ILD are insufficient. Thus, we cannot evaluate treatment correctly.
The prevalence of ILD with all-stage LC is poorly evaluated (1). In a limited group of patients, the prevalence of ILD with LC has been reported. Previous studies showed that among primary LC patients treated by surgical resection, $5.8 \%$ to $16.8 \%$ had underlying ILD as detected by histopathology of the resected specimen $(2,3)$. Other studies reported that $7.3 \%$ to $24.3 \%$ of small cell lung cancer (SCLC) or non-small cell lung cancer (NSCLC) patients who had been treated with chemotherapy had ILD (4-7). However, no published studies have revealed the prevalence of ILD with all stages of LC and all treatments, including best supportive care (BSC). 


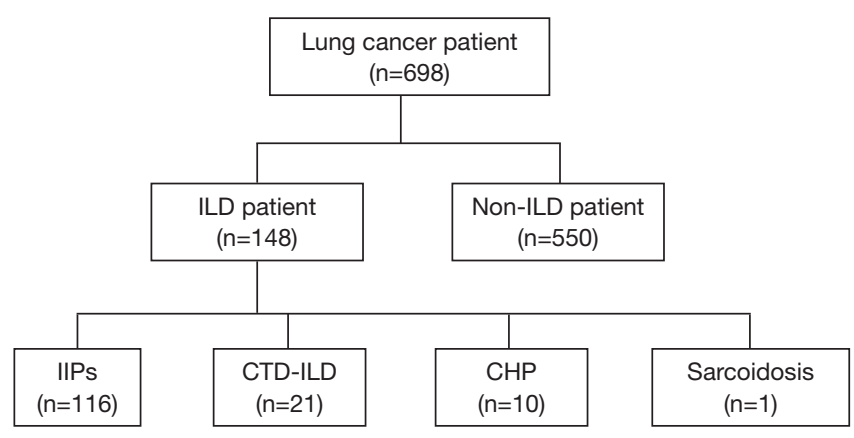

Figure 1 Diagram showing the relationship between all lung cancer patient and lung cancer patient with ILD and non-ILD. ILD, interstitial lung disease; IIPs, idiopathic interstitial pneumonias; CTD, connective tissue disease; CHP, chronic hypersensitivity pneumonitis.

Clinical information regarding treatment is minimal, and no study has focused on BSC in LC-ILD patients. A previous study (8) compared idiopathic interstitial pneumonias (IIPs) patient who received chemotherapy with IIPs patients who received BSC. However, this study didn't report characteristics of BSC patients. We can't evaluate the efficacy of therapy for LC-ILD patients, because it is necessary to evaluate $\mathrm{BSC}$ patients who can receive treatment. We need the data of BSC patients who decline treatment. This study aimed to reveal the prevalence of ILD among all stages of LC and real-world treatment data, including BSC, in LC-ILD patients.

\section{Methods}

This study was performed at a single centre. Patients with LC who were admitted to Tokyo Medical and Dental University Hospital during the 5-year period from January 1, 2013, to December 31, 2017, were retrospectively analysed. In this study, BSC was defined therapies to relief from diseaserelated symptoms other than systemic anti-cancer therapy (9). CT of the chest was evaluated by two pulmonologists. When their diagnosis differed, two other pulmonologists reviewed the CT. If their diagnoses differed, the case was discussed until a diagnosis was reached. We classified ILD into two groups: UIP pattern and non-UIP pattern. The UIP pattern was defined as a predominant subpleural reticular abnormality with honeycombing. In addition, it was necessary for the absence of any features of upper lobe or peribronchovascular predominance, predominant ground-glass abnormality or micronodules (10).
Diagnoses of AE of ILD were made in accordance with the following criteria: worsening of dyspnoea within 30 days; new radiologic bilateral ground-glass abnormality and/or consolidation superimposed on a background of reticular shadows or honeycombing; no evidence of pulmonary infection and exclusion of alternate causes, including left ventricle heart failure, pulmonary embolism, and acute lung injury of identifiable cause; and less than four weeks interval between the last round of therapy and $\mathrm{AE}$ onset $(11,12)$.

This study was approved by the institutional review board at Tokyo Medical and Dental University (approval numbers: M2017-281).

\section{Statistical analysis}

In patients who received therapy, overall survival (OS) was defined as the time between the therapy onset and the date of death from any cause. In patients who received chemotherapy after developing postoperative recurrence, OS was defined as the time between the date of the start of chemotherapy and death from any cause. In patients who received BSC only, OS was defined as the time between the date of diagnosis and death from any cause. The observation period was until May 31, 2018. OS was analysed using the Kaplan-Meier method. Differences in clinical data between two independent samples were tested using the Mann-Whitney U test or Student's $t$-test, as appropriate. All $\mathrm{P}$ values are reported as two-sided, and values $<0.05$ considered statistically significant. All statistical analyses were performed with EZR (Saitama Medical Center, Jichi Medical University, Saitama, Japan) (13).

\section{Results}

\section{Patient characteristics}

In total, 698 patients with LC (6 patients had two LC) were identified for this study. Of 698 patients, 148 (21.2\%) suffered from LC with ILD (Figure 1). Fifty-nine patients attended our hospital with ILD before LC diagnosis. Relevant characteristics with or without ILD are shown in Table 1. Patients with ILD were significantly older, more often male and smokers, had a higher serum Krebs von den Lungen-6 (KL-6) level, which is a serum marker for interstitial pneumonia, and a lower predicted percent volume capacity (\%VC) than those without ILD. The frequency of adenocarcinoma was lower, and squamous cell carcinoma and small cell carcinoma were higher in patients with ILD than in 
Table 1 Characteristics of lung cancer patients with or without ILD

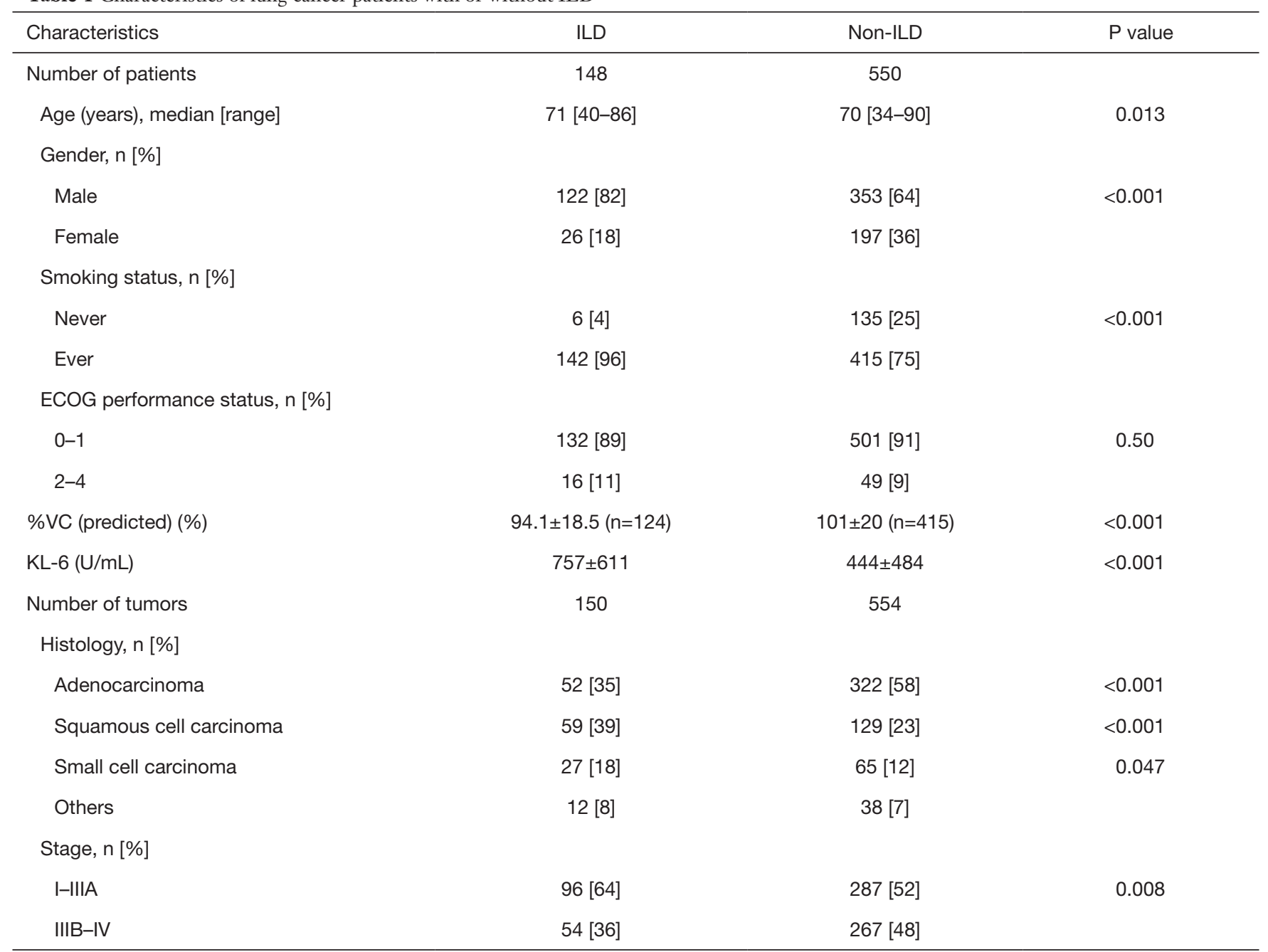

Data are presented by mean \pm standard deviation. ILD, interstitial lung disease; ECOG, Eastern Cooperative Oncology Group; \%VC, percent vital capacity; KL-6, Krebs von der Lungen-6.

those without ILD. Advanced (stage IIIB-IV) cancer was less prevalent in patients with ILD than in those without ILD.

Most patients suffered from IIPs (116 patients, 78\%). Twenty-one patients (14\%) suffered from connective tissue disease-associated interstitial pneumonia, and in some patients, connective tissue diseases overlapped. Thirteen patients had rheumatoid arthritis, 6 had systemic sclerosis, 3 had Sjögren's syndrome, 2 had polymyositis and dermatomyositis, and one patient had systemic lupus erythematosus. Chronic hypersensitivity pneumonitis patients (10 patients, 7\%) were few. Sarcoidosis patients was the only one. The UIP pattern of ILD was observed in 69 patients $(47 \%)$, whereas non-UIP was observed in 79 patients $(53 \%)$. Seventeen patients $(11 \%)$ used home oxygen therapy. Thirty-one patients (21\%) used steroids or immunosuppressants. Two patients (1\%) used antifibrotics.

\section{Characteristics of LC-ILD patients who received chemotherapy and BSC}

Of 148 LC-ILD patients, 71 (48\%) received chemotherapy including postoperative recurrence, 41 patients $(28 \%)$ received surgery or surgery plus adjuvant chemotherapy, 20 patients (14\%) received BSC only, and 16 patients (11\%) received other treatments. In BSC patients, 11 patients whom physicians thought could receive therapy declined treatment after being informed of the benefits and risks of treatment. All of them were recommended chemotherapy. 
Table 2 Combined lung cancer with ILD patients with chemotherapy and BSC only because of declining treatment

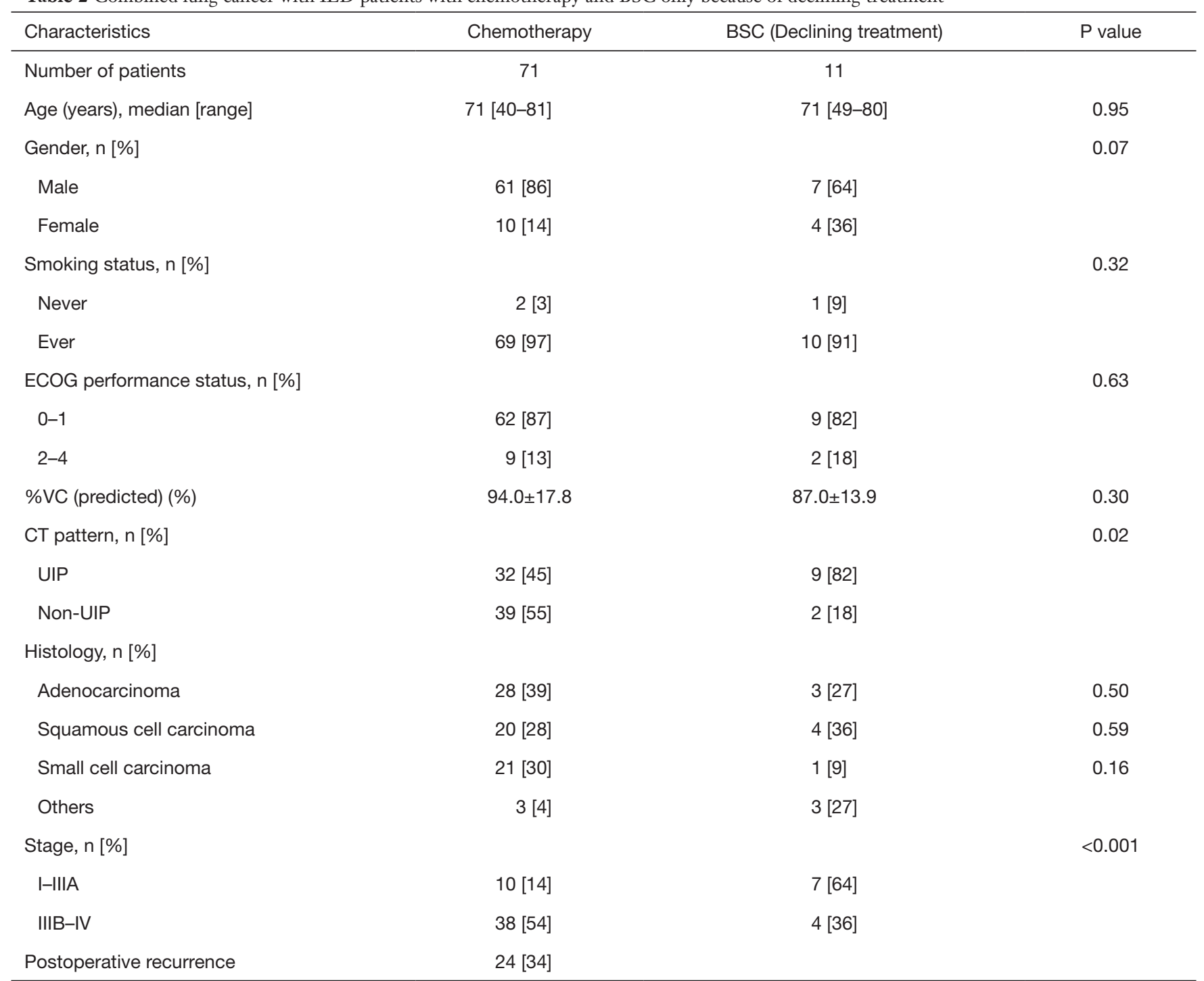

Data are presented by mean \pm standard deviation. One chemotherapy patient had both adeno and squamous lung cancer. ILD, Interstitial lung disease; BSC, best supportive care; ECOG, Eastern Cooperative Oncology Group; \%VC, percent vital capacity; UIP, usual interstitial pneumonia.

The reasons for refusing treatment were that 3 patients thought chemotherapy was only a palliative therapy, 3 patients thought chemotherapy of LC-ILD patients was high-risk, one patient had no symptoms from LC, and there were no records of the reasons for the other 4 patients. The reasons for BSC in 9 other patients were related to being elderly (5 patients), having low pulmonary function or severe ILD with a shorter prognosis than LC (3 patients), and poor performance status (1 patient).

The characteristics of LC-ILD patients who received chemotherapy (one patient had both adeno and squamous
LC) and LC-ILD patients who received BSC only because of declining treatment are shown in Table 2. In chemotherapy patients, NSCLC occurred in 50 (70\%) patients, whereas SCLC occurred in 21 (30\%). Ten patients who received chemotherapy suffered from early stage LC (stage I-IIIA), 37 patients had advanced-stage LC, and 24 patients had postoperative recurrence. Patients who suffered from early-stage LC received chemotherapy within 45 days of LC diagnosis. The reason for receiving chemotherapy in spite of early stage LC was that lung function in 5 patients was too poor to receive surgery, 4 patients suffered from 


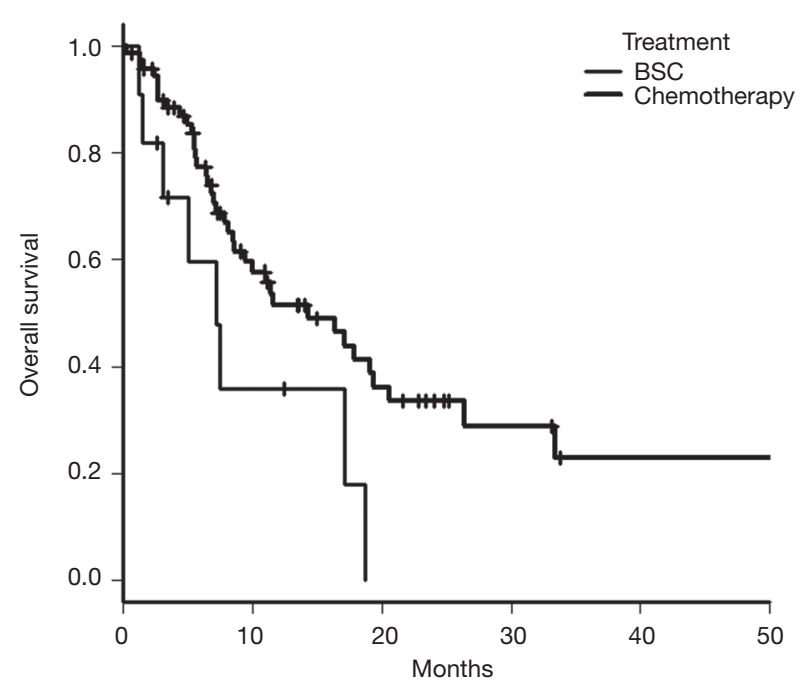

Figure 2 Overall survival time combined lung cancer and interstitial lung disease patients who received chemotherapy and who received best supportive care (BSC) only because of declining treatment (median 14.3 vs. 7.2 months, $\mathrm{P}=0.036$ ).

SCLC, and one patient refused surgery.

In BSC patients who declined treatment, the frequency of UIP patterns was higher than that of non-UIP pattern. Almost all patients suffered from NSCLC. Early stage LC (stage IIIIA) was observed in 7 (64\%) patients, and advanced-stage LC (stage IIIB-IV) was observed in $4(36 \%)$ patients.

There was no difference in age, gender, smoking status, ECOG performance status, \%VC, histology of LC, clinical stage between chemotherapy patients and BSC patients who declined treatment. BSC patients who declined treatment had a higher percentage of cases with UIP pattern and early stage cancer than chemotherapy patients.

There were no difference between chemotherapy of LCILD patients with UIP pattern and with non-UIP pattern in histology, $1^{\text {st }}$-line regimen, and number of regimens (Table S1). There were 9 connective tissue disease-associated interstitial pneumonia patients who received chemotherapy. All of them were received platinum-based chemotherapy as $1^{\text {st }}$-line treatment. There were 2 chronic hypersensitivity pneumonitis patients who received chemotherapy. They were received S-1 as $1^{\text {st }}$-line treatment.

\section{The survival time of $L C-I L D$ patients who received chemotherapy and BSC}

The survival curve of LC-ILD patients who received chemotherapy and who received BSC only because of declining treatment is shown in Figure 2. The median survival time of all LC-ILD patients who received chemotherapy was 14.3 months (95\% confidence interval, 8.5-19.4 months). The median survival time of BSC patients who declined treatment was 7.2 months. The median survival time of patients who received chemotherapy was longer than that of only BSC patients who declined treatment $(\mathrm{P}=0.036)$.

\section{LC patients who received chemotherapy with ILD or without ILD}

The characteristics of LC patients who received chemotherapy with ILD were different from those of LC patient who received chemotherapy without ILD in histology and stage (Table S2).

The median survival time of advanced stage (IIIB-IV) and postoperative recurrence NSCLC patients without ILD who received chemotherapy was longer than that of NSCLC patients with ILD who received chemotherapy (34.3 vs. 16.4 months, $\mathrm{P}=0.004$ ). The median survival time of advanced stage (IIIB-IV) and postoperative recurrence SCLC patients without ILD who received chemotherapy was no statistically significant different from that of SCLC patients with ILD who received chemotherapy (16.9 vs. 8.1 months, $\mathrm{P}=0.18)$.

\section{Treatment-related AE of ILD}

Treatment-related AE of ILD was diagnosed in 15 of 115 patients (13\%). Treatment-related AE of ILD developed in 2 of 36 patients $(6 \%)$ in the surgery-only group and in 13 of 78 patients (17\%) in the chemotherapy group, including postoperative recurrence, chemoradiotherapy and adjuvant chemotherapy. Of 15 patients, 13 patients $(87 \%)$ were suffered from IIPs, and the other 2 patients $(13 \%)$ were suffered from chronic hypersensitivity pneumonia. The UIP pattern of ILD was observed in 8 patients (53\%), whereas non-UIP was observed in 7 patients (47\%). One patient who suffered from IPF used $10 \mathrm{mg} /$ day prednisolone for ILD, and one patient who suffered from chronic hypersensitivity pneumonitis whose CT pattern was UIP pattern used $11 \mathrm{mg} /$ day prednisolone for ILD. The others didn't receive therapy against ILD.

The incidence of AE of ILD caused by platinumbased regimens was 6 patients ( $46 \%$ of patients caused by chemotherapy). Ten patients suffered from treatment-related $\mathrm{AE}$ of ILD in the $1^{\text {st }}$-line, including maintenance therapy, 
2 patients were receiving $3^{\text {rd }}$-line chemotherapy, and one patient was in adjuvant chemotherapy. Approximately half of the patients (53\%) survived, and approximately half died from $\mathrm{AE}$ of ILD (47\%). The median survival time from the start of therapy to death from any cause was 7.0 months $(95 \%$ confidence interval, 2.1-9.5 months). The 1-year survival probability was calculated as $22 \%$.

\section{Discussion}

We revealed the prevalence of ILD and LC co-occurrence and real-world BSC treatment data in LC-ILD patients. Two main findings are indicated. One important finding is that the presence of ILD in patients with LC is common. The second finding is that chemotherapy for LC-ILD patients is effective.

In the present study, the prevalence of ILD with LC was $21.2 \%$. The rate is comparable with previous studies (2-7) (5.8\% to $24.3 \%)$. Most previous studies focused on a limited group of patients, and excluded patients who received only BSC or whose ILD was not idiopathic pulmonary fibrosis. The proportion of patients who received BSC only was not small $(6-8,14)$, and in chronic hypersensitivity pneumonitis or connective tissue disease-associated ILD patients, LC is not rare $(15,16)$. This study included 53 patients who received only BSC (20 with and 33 without ILD) and 32 patients whose ILD was not IIPs (connective tissue diseaseassociated interstitial pneumonia or chronic hypersensitivity pneumonitis or others). If we exclude patients who received only BSC, the prevalence of ILD with LC is $19.8 \%$. If we exclude patients whose ILD was not IIP, the prevalence of ILD with LC is $17.4 \%$.

Previous studies showed ILD including pre-clinical or asymptomatic interstitial lung abnormalities was associated with an increased risk of LC (1,17-20). There are many possible underlying mechanisms linking LC and ILD (1). IPF conveys a five-fold increased risk of LC after adjusting for smoking (18), and the 5-year cumulative incidence rate of LC in IPF patient is $12.2 \%$ to $15.4 \%(19,20)$. Approximately one-third of LC-ILD patients in our study attended our hospital before receiving a diagnosis of LC. Most of the papers about LC-ILD did not report the number of patients who attended a hospital with ILD before the diagnosis of LC. There were a little number of patients who received therapy for ILD in this study. We couldn't tell ILD therapy is beneficial for LC-ILD patients. The clinical trial of carboplatin plus nanoparticle albuminbound paclitaxel with or without nintedanib for patients with LC and IPF started. This would provide the beneficial information about it (21).

To the best of our knowledge, the present study is the first to fully report LC-ILD patients who received BSC only. A previous study (8) compared IIPs patient who received chemotherapy with IIPs patients who received BSC. The definition of BSC in the study was equivalent to that in our study. However, this study didn't report characteristics of BSC patients, so it was impossible to evaluate true benefit of chemotherapy. So, we carried out the investigation about LC-ILD patients who received BSC only. In our study, more than half of BSC patients declined treatment after being informed of the benefits and risks of treatment. All BSC patients who declined treatment were recommended chemotherapy by physicians. Their characteristics was similar to chemotherapy patients' characteristics. We cannot strictly discuss the efficacy of therapy for LC-ILD patients because their characteristics of CT pattern and stage were different in BSC patients and chemotherapy patients, and the number of BSC patients whom physicians thought could receive treatment was small. However, the median survival time of patients who received chemotherapy was longer than that of BSC-only patients who declined treatment. Therefore, it is possible that chemotherapy for LC-ILD patients is effective. More data should be gathered.

The present study has several limitations. First, this study is a retrospective single-centre study. A prospective multicentre large study is desirable to further investigate the prevalence of ILD with LC and treatment. Second, our study excluded patients who were not pathologically or cytologically proven to have LC. Even if a physician detects a lesion which is suspected LC with X-ray or CT, he does not use bronchoscopy, needle biopsy or surgical biopsy for patients who have severe ILD or poor respiratory function. Our study has the possibility of excluding these patients. Therefore, we may have underestimated the prevalence of ILD with LC. Third, LC-ILD patients in our study have better lung function than in the previous studies $(4,14,22)$. It might influence prevalence and survival results. There is a possibility that the lung function data in our study excluded the data of bad lung function. In our study, pulmonary function tests data were not available in 24 LC-ILD patients. Of 24 patients, 9 patients had bad performance status, ECOG performance status 2-4. Fourth, the number of BSC patients who declined treatment is only 11. Considering the complexity of pathogenesis, diagnosis and therapy of LC and ILD, 11 cases is very limited. 
In conclusion, LC patients with ILD are not rare, and there is a possibility that treatment for LC-ILD patients is beneficial. We should plan a clinical study or trial for LC patients with ILD.

\section{Acknowledgments}

We thank all members of the Department of Respiratory Medicine of Tokyo Medical and Dental University.

\section{Footnote}

Conflicts of Interest: The authors have no conflicts of interest to declare.

Ethical Statement: The authors are accountable for all aspects of the work in ensuring that questions related to the accuracy or integrity of any part of the work are appropriately investigated and resolved. This study was approved by the institutional review board at the Tokyo Medical and Dental University (approval numbers: M2017281) and we waived the requirement for patient consent because of the retrospective nature of this study.

\section{References}

1. Naccache JM, Gibiot Q, Monnet I, et al. Lung cancer and interstitial lung disease: a literature review. J Thorac Dis 2018;10:3829-44.

2. Omori T, Tajiri M, Baba T, et al. Pulmonary Resection for Lung Cancer in Patients With Idiopathic Interstitial Pneumonia. Ann Thorac Surg 2015;100:954-60.

3. Sato T, Teramukai S, Kondo H, et al. Impact and predictors of acute exacerbation of interstitial lung diseases after pulmonary resection for lung cancer. J Thorac Cardiovasc Surg 2014;147:1604-1611.e3.

4. Watanabe N, Taniguchi H, Kondoh Y, et al. Chemotherapy for extensive-stage small-cell lung cancer with idiopathic pulmonary fibrosis. Int J Clin Oncol 2014;19:260-5.

5. Togashi Y, Masago K, Handa T, et al. Prognostic significance of preexisting interstitial lung disease in Japanese patients with small-cell lung cancer. Clin Lung Cancer 2012;13:304-11.

6. Kanaji N, Tadokoro A, Kita N, et al. Impact of idiopathic pulmonary fibrosis on advanced non-small cell lung cancer survival. J Cancer Res Clin Oncol 2016;142:1855-65.

7. Watanabe N, Taniguchi H, Kondoh Y, et al. Efficacy of chemotherapy for advanced non-small cell lung cancer with idiopathic pulmonary fibrosis. Respiration 2013;85:326-31.

8. Kashiwabara K, Semba H, Fujii S, et al. Difference in benefit of chemotherapy between small cell lung cancer patients with interstitial pneumonia and patients with nonsmall cell lung cancer. Anticancer Res 2015;35:1065-71.

9. Lester JF, Agulnik J, Akerborg O. What constitutes best supportive care in the treatment of advanced non-small cell lung cancer patients?--Results from the lung cancer economics and outcomes research (LUCEOR) study. Lung Cancer 2013;82:128-35.

10. Raghu G, Rochwerg B, Zhang Y, et al. An Official ATS/ ERS/JRS/ALAT Clinical Practice Guideline: Treatment of Idiopathic Pulmonary Fibrosis. An Update of the 2011 Clinical Practice Guideline. Am J Respir Crit Care Med 2015;192:e3-19.

11. Kondoh Y, Taniguchi H, Kawabata Y, et al. Acute exacerbation in idiopathic pulmonary fibrosis. Analysis of clinical and pathologic findings in three cases. Chest 1993;103:1808-12.

12. Akira M, Hamada H, Sakatani M, et al. CT findings during phase of accelerated deterioration in patients with idiopathic pulmonary fibrosis. AJR Am J Roentgenol 1997;168:79-83.

13. Kanda Y. Investigation of the freely available easy-touse software 'EZR' for medical statistics. Bone Marrow Transplant 2013;48:452-8.

14. Isobe K, Hata $Y$, Sakamoto S, et al. Clinical characteristics of acute respiratory deterioration in pulmonary fibrosis associated with lung cancer following anti-cancer therapy. Respirology 2010;15:88-92.

15. Kuramochi J, Inase N, Miyazaki Y, et al. Lung cancer in chronic hypersensitivity pneumonitis. Respiration 2011;82:263-7.

16. Watanabe S, Saeki K, Waseda Y, et al. Lung cancer in connective tissue disease-associated interstitial lung disease: clinical features and impact on outcomes. J Thorac Dis 2018;10:799-807.

17. Whittaker Brown SA, Padilla M, Mhango G, et al. Interstitial Lung Abnormalities and Lung Cancer Risk in the National Lung Screening Trial. Chest 2019. [Epub ahead of print].

18. Le Jeune I, Gribbin J, West J, et al. The incidence of cancer in patients with idiopathic pulmonary fibrosis and sarcoidosis in the UK. Respir Med 2007;101:2534-40.

19. Kato E, Takayanagi N, Takaku Y, et al. Incidence and predictive factors of lung cancer in patients with idiopathic 
pulmonary fibrosis. ERJ Open Res 2018;4:00111-2016.

20. Ozawa Y, Suda T, Naito T, et al. Cumulative incidence of and predictive factors for lung cancer in IPF. Respirology 2009; 14:723-8.

21. Otsubo K, Kishimoto J, Kenmotsu H. Treatment Rationale and Design for J-SONIC: A Randomized Study of Carboplatin Plus Nab-paclitaxel With or Without Nintedanib for Advanced Non-Small-cell Lung Cancer

Cite this article as: Kawahara T, Sakashita H, Suzuki T, Tateishi T, Miyazaki Y. Real world data of combined lung cancer and interstitial lung disease. J Thorac Dis 2019;11(10):41444151. doi: $10.21037 /$ jtd.2019.10.01
With Idiopathic Pulmonary Fibrosis. Clin Lung Cancer 2018;19:e5-9.

22. Yasuda Y, Hattori Y, Tohnai R, et al. The safety and efficacy of carboplatin plus nanoparticle albumin-bound paclitaxel in the treatment of non-small cell lung cancer patients with interstitial lung disease. Jpn J Clin Oncol 2018;48:89-93. 
Supplementary

Table S1 Chemotherapy of LC-ILD patients with UIP pattern with non-UIP pattern

\begin{tabular}{|c|c|c|c|}
\hline $\begin{array}{l}\text { Histology and characteristics } \\
\text { of chemotherapy }\end{array}$ & UIP & Non-UIP & $P$ value \\
\hline Number of patients & 32 & 39 & \\
\hline \multicolumn{4}{|l|}{ Histology, n [\%] } \\
\hline Adenocarcinoma & 12 [36] & $16[41]$ & 0.69 \\
\hline Squamous cell carcinoma & $9[27]$ & $11[28]$ & 0.94 \\
\hline Small cell carcinoma & 10 [33] & $11[28]$ & 0.85 \\
\hline Others & $2[6]$ & $1[3]$ & \\
\hline Number of NSCLC patients & 22 & 28 & \\
\hline \multicolumn{4}{|l|}{ First-line regimen, $\mathrm{n}[\%]$} \\
\hline $\mathrm{CBDCA}+\mathrm{NabPTX}$ & $14[64]$ & $16[57]$ & 0.65 \\
\hline $\mathrm{CBDCA}+\mathrm{S}-1$ & $1[5]$ & $3[11]$ & 0.44 \\
\hline CBDCA + PTX & $3[14]$ & $1[4]$ & 0.21 \\
\hline S-1 & 2 [9] & $2[7]$ & 0.82 \\
\hline Others & 2 [9] & $6[21]$ & \\
\hline \multicolumn{4}{|l|}{ Number of regimens [\%] } \\
\hline One & 13 [59] & 12 [43] & 0.26 \\
\hline Two & $5[23]$ & 7 [25] & 0.86 \\
\hline Three or more & $4[18]$ & 9 [32] & 0.27 \\
\hline Number of SCLC patients & 10 & 11 & \\
\hline \multicolumn{4}{|l|}{ First-line regimen, $\mathrm{n}$ [\%] } \\
\hline CBDCA + ETP & $7[70]$ & $8[73]$ & 0.93 \\
\hline CDDP + ETP & $2[20]$ & $3[27]$ & 0.74 \\
\hline Others & $1[10]$ & 0 & \\
\hline \multicolumn{4}{|l|}{ Number of regimens } \\
\hline One & $5[50]$ & $4[36]$ & 0.57 \\
\hline Two & $3[30]$ & $4[36]$ & 0.80 \\
\hline Three or more & $2[20]$ & $3[27]$ & 0.74 \\
\hline
\end{tabular}

One UIP pattern patient had both adeno and squamous lung cancer. LC, lung cancer: ILD, Interstitial lung disease; UIP, usual interstitial pneumonia; NSCLC, non-small cell lung cancer; CBDCA ,carboplatin; NabPTX, nanoparticle albumin-bound paclitaxel; PTX, paclitaxel; SCLC, small cell lung cancer: ETP, etoposide; CDDP, cisplatin.
Table S2 Characteristics of lung cancer patients who received chemotherapy with or without ILD

\begin{tabular}{|c|c|c|c|}
\hline Characteristics & ILD & non-ILD & $P$ value \\
\hline Number of patients & 71 & 247 & \\
\hline Age (years), median [range] & $71[40-81]$ & 68 [34-87] & 0.06 \\
\hline Gender, n [\%] & & & $<0.001$ \\
\hline Male & $61[86]$ & $151[61]$ & \\
\hline Female & $10[14]$ & 96 [39] & \\
\hline Smoking status, n [\%] & & & $<0.001$ \\
\hline Never & $2[3]$ & 70 [28] & \\
\hline Ever & 69 [97] & $177[72]$ & \\
\hline ECOG performance status, $\mathrm{n}$ [\%] & & & 0.41 \\
\hline $0-1$ & $62[87]$ & 224 [91] & \\
\hline $2-4$ & 9 [13] & $23[9]$ & \\
\hline$\%$ VC (predicted) (\%) & $94.0 \pm 17.8$ & $97.6 \pm 18.8$ & 0.2 \\
\hline \multicolumn{4}{|l|}{ Histology, n [\%] } \\
\hline Adenocarcinoma & 28 [39] & $162[66]$ & $<0.001$ \\
\hline Squamous cell carcinoma & 20 [28] & $40[16]$ & 0.03 \\
\hline Small cell carcinoma & $21[30]$ & $35[14]$ & 0.003 \\
\hline Others & $3[4]$ & $10[4]$ & \\
\hline Stage, n [\%] & & & 0.02 \\
\hline I-IIIA & $10[14]$ & $14[6]$ & \\
\hline IIIB-IV & $38[54]$ & $173[70]$ & \\
\hline Postoperative recurrence, $\mathrm{n}[\%]$ & 24 [34] & 60 [24] & \\
\hline
\end{tabular}

One UIP pattern patient had both adeno and squamous lung cancer. Data are presented by mean \pm standard deviation. ILD, interstitial lung disease; ECOG, Eastern Cooperative Oncology Group; \%VC, percent vital capacity. 